\title{
A pH Sensor Based on Corrugated Long-Period Fiber Grating
}

\author{
Chia-Chin Chiang, Cheung-Hwa $\mathrm{Hsu}^{1,{ }^{*}}$ and Chao-Hui Ou${ }^{1}$ \\ Department of Mechanical Engineering, National Kaohsiung University of Applied Sciences, \\ 415 Chien Kung Road, Kaohsiung 807, Taiwan \\ 'Department of Mold and Die Engineering, National Kaohsiung University of Applied Science, \\ 415 Chien Kung Road, Kaohsiung 807, Taiwan
}

(Received November 5, 2012; accepted February 4, 2013)

Key words: corrugated long-period fiber grating, optical fiber sensor, $\mathrm{pH}$

In the present study, we propose a $\mathrm{pH}$ sensor based on a corrugated long-period fiber grating (CLPFG). CLPFG is highly sensitive to refractive index changes, and, with an appropriate sensitive coating, can detect a variety of chemical parameters. CLPFG is designed to act as a spectral loss device that couples with a discrete wavelength out of the optical fiber as a function of the surrounding refractive index. By applying a special coating that changes the refractive index through the absorption of target molecules to the CLPFG surface, the CLPFG can become a transducer for chemical measurement. In this paper, the incorporation of a polyvinyl alcohol and polycyclic acid (PVA-PAA) coating onto CLPFG to produce an optical-fiber-based $\mathrm{pH}$ sensor with a $\mathrm{pH}$ detection range from 2 to 6 is discussed.

\section{Introduction}

A long-period fiber grating (LPFG) has been applied in the development of optical sensing systems, ${ }^{(1)}$ and has increasingly been applied in sensing for strain, ${ }^{(1)}$ temperature, ${ }^{(2)}$ and refractive indices ${ }^{(3-4)}$ and $\mathrm{pH} .{ }^{(5-6)}$ LPFGs promote coupling between the propagating core and cladding modes, providing an attenuation loss band, which can be used for sensor applications. In 2009, Chiang et al.(7) reported on the use of a sandwiched SU-8 thick photoresist technique for the fabrication of a corrugated longperiod fiber grating (CLPFG) for potential applications as a sensor. CLPFG sensors possess various advantages over conventional sensors, such as small dimensions, light weight, good sensitivity, good long-term stability, corrosion resistance, and resistance to magnetic and electromagnetic interference.

${ }^{*}$ Corresponding author: e-mail: chhsu@kuas.edu.tw 
The CLPFG sensor element is highly sensitive to refractive index changes and, with the appropriate coatings, can be designed to be sensitive to a variety of chemical parameters. In this study, the feasibility and performance of the sensor were investigated through the application of unique $\mathrm{pH}$-sensitive hydrogel coatings to the CLPFG sensor element.

\section{Materials and Methods}

\subsection{CLPFG refractive index calibration}

The CLPFG was fabricated on dispersion-shifted fiber (DSF) using lithography and etching processes to form the grating pattern. Figure 1 shows the schematic diagram of the characterization measurement of the CLPFG. This measurement system consisted of a superluminescent laser diode (SLD), an optical spectrum analyzer (OSA), and a precise linear stage. When the force loading was applied on the CLPFG, the refractive index of the core generated a periodic distribution along the longitudinal axis of the fiber to obtain the characteristics of the CLPFG.

The CLPFG was submerged in a selected refractive index fluid, and the spectra were recorded using OSA. After each measurement was recorded, the CLPFG was cleaned thoroughly until the spectral response returned to the original state.

\subsection{Coating process}

Polymer gel preparation was the first step in the coating process. Ethyl acetate is primarily used as a solvent and diluent because of its low cost, low toxicity, and agreeable odor. Conversely, it is rarely selected as a reaction solvent because it is prone to hydrolysis and transesterification. It is also very volatile and has a low boiling point. Owing to these properties, it can be removed from a sample by heating the sample in a hot water bath and providing ventilation with compressed air. Thus, ethyl acetate was

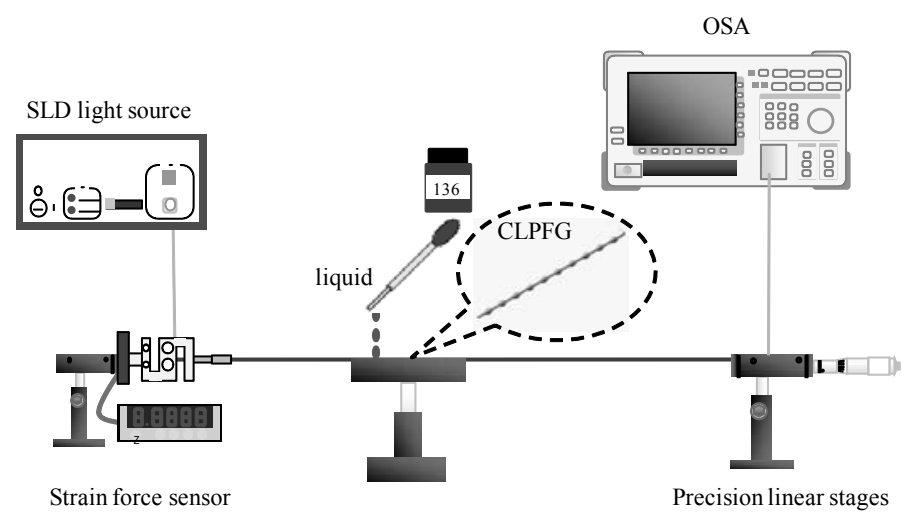

Fig. 1. Schematic of the characterization measurement of CLPFG. 
used to mix polyvinyl alcohol (PVA) with polyacrylic acid (PAA); the related schematic coating process is shown in Fig. 2. The polymer gel was made by using $80 \%$ PVA and $20 \%$ PAA.

More specifically, PVA with an average molecular weight of 78000 (Polysciences, Inc.) and PAA with an average molecular weight of 90000 were dissolved in ethyl acetate solvent in relative proportions of 80 and $20 \%$ by weight, respectively. A CLPFG was first vertically inserted into, then vertically removed from this polymer solution at a speed of $10 \mathrm{~mm} / \mathrm{s}$. After that, the CLPFG was dried using a heater at $80{ }^{\circ} \mathrm{C}$ for $20 \mathrm{~min}$. Next, the coating of the CLPFG was thermally crosslinked at $130{ }^{\circ} \mathrm{C}$ for $60 \mathrm{~min}$. After the coating, the CLPFG sensor was allowed to soak for $24 \mathrm{~h}$ in buffer solutions with $\mathrm{pH}$ ranging from 2 to 8 . Finally, the sensor measured the $\mathrm{pH}$ of the buffer solution.

\section{Results and Discussion}

The CLPFG exhibits the resonant dip spectrum for a particular wavelength. When CLPFG is subjected to mechanical forces or external environmental change, such as changes in refractive index or temperature, the transmission spectra are changed through wavelength shift or resonance attenuation loss. Figure 3 shows the characterization of CLPFGs with various force loadings. The transmission loss of the resonant dip in the spectrum changes with the loading. Furthermore, when there is no loading, no resonant dip will be observed. Consequently, the wavelength variation is a better candidate for the $\mathrm{pH}$ sensing.

For the refractive index calibration test, the CLPFG was submerged in the refractive index standards. There are no data points above 1.5 because the CLPFG spectral dip would disappear above this refractive index. This is due to the characteristics of the long-period grating. Figure 4 shows a plot of the spectral loss dips that occur as a result of immersing the CLPFG in the various refractive index standards. The resolution of the OSA used for this test was $0.2 \mathrm{~nm}$. By tracking the spectral loss dip, the CLPFG can be used to measure refractive index changes in the surrounding medium.

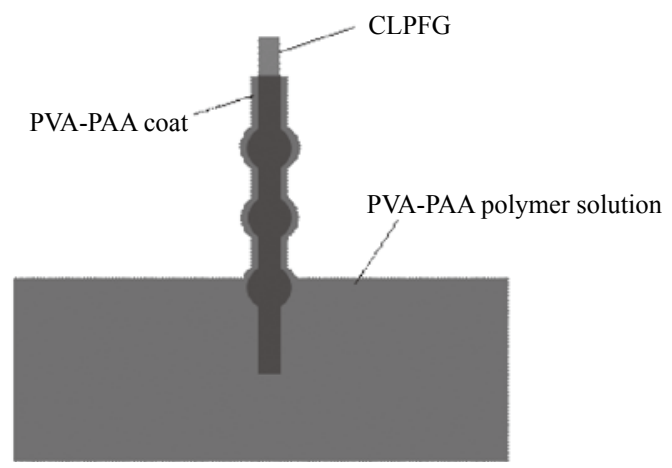

Fig. 2. Schematic of the coating process. 


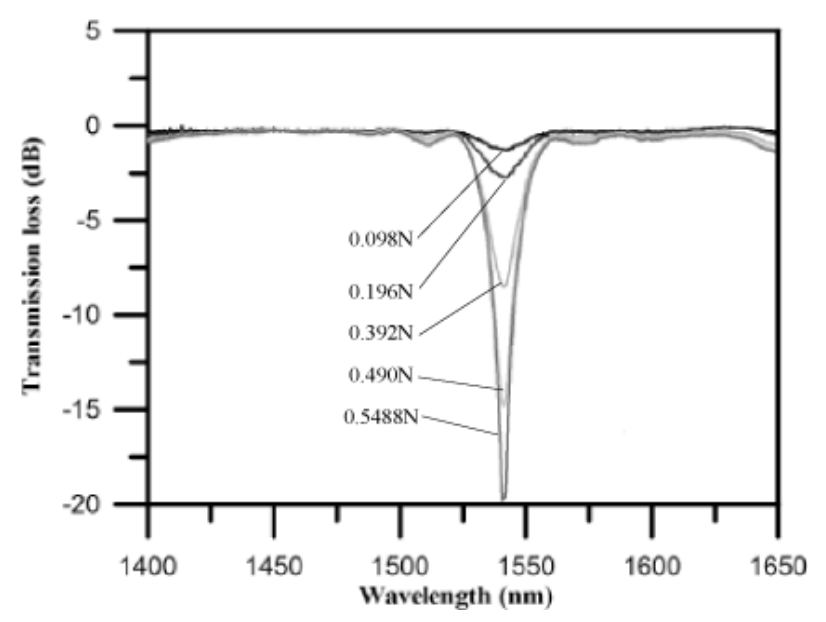

Fig. 3. Spectra of CLPFGs with various force loadings.

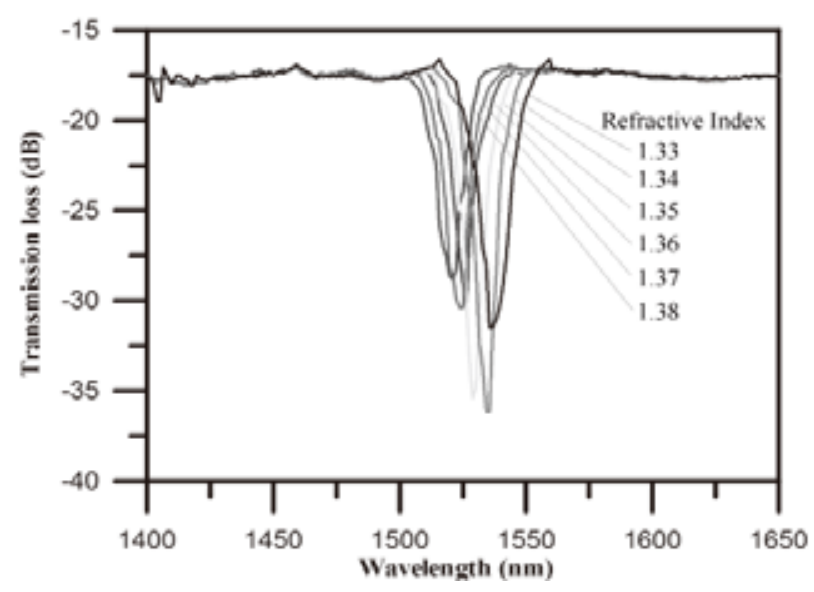

Fig. 4. Shifts in the spectra of CLPFG with refractive index changes.

As shown in Fig. 5, when the refractive index was increased, the wavelength decreased. The CLPFG response had its highest sensitivity to the refractive index changes between 1.4 and 1.5. This information is critical for developing sensors with increased sensitivity to a desired environmental parameter. When the refractive index of the solution is above 1.5 , it will be too close to the refractive index of the fiber cladding and the spectral loss dip will disappear.

To test its ability to sense $\mathrm{pH}$, the CLPFG coated with PVA-PAA was immersed in the various buffer solutions with $\mathrm{pH}$ levels ranging from 2 to 8 , and the resultant changes in the spectral response were observed. As shown in Fig. 6, the resonant dip 


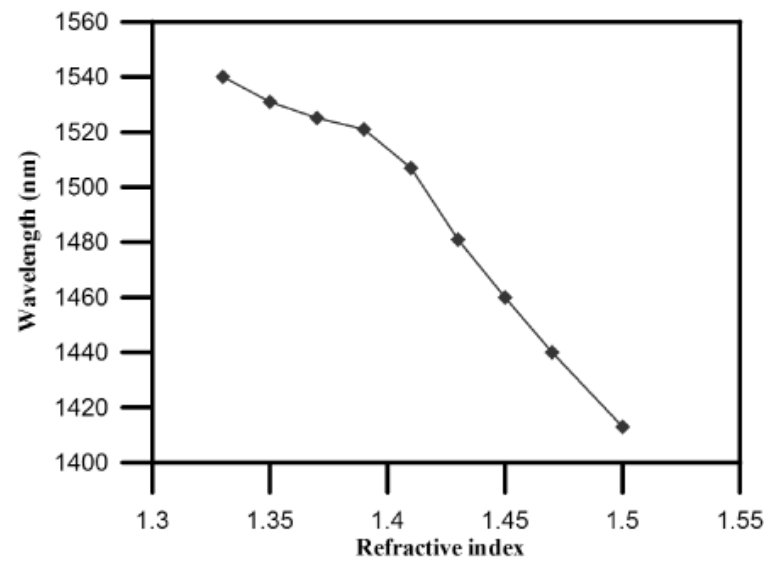

Fig. 5. Wavelength shift of CLPFG with various refractive indexes.

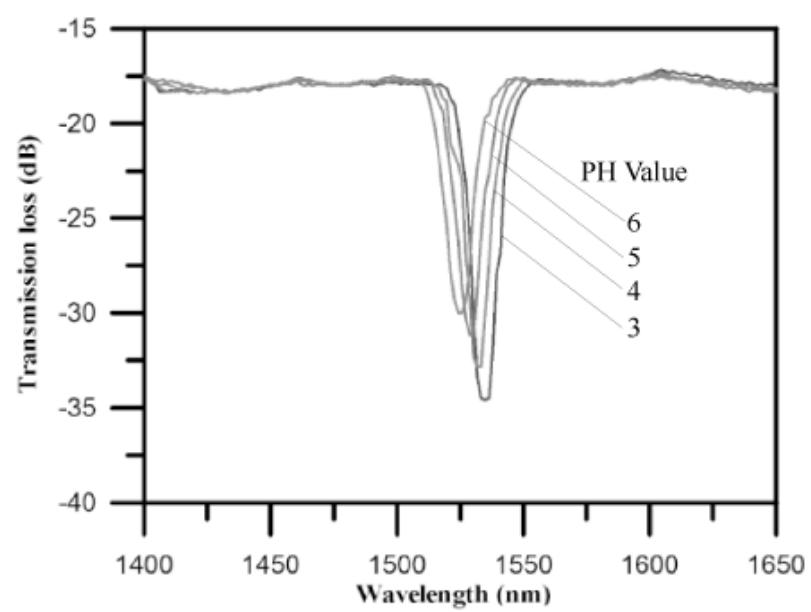

Fig. 6. Spectra of the PVA-PAA-coated CLPFG with various $\mathrm{pH}$ solutions.

wavelength of the $\mathrm{pH}$ sensor shifted with various $\mathrm{pH}$ levels. Figure 7 shows a plot of $\mathrm{pH}$ versus wavelength from the spectra. According to this plot, the wavelength shift of the $\mathrm{pH}$ sensor was sensitive between $\mathrm{pHs} 2$ and 6 . However, the wavelength shift of the $\mathrm{pH}$ sensor was negligible between pHs 6 and 8. The experimental results show that the resonant wavelength shifted $10.5 \mathrm{~nm}$ between $\mathrm{pHs} 2$ and 6; accordingly, the sensitivity of the $\mathrm{pH}$ sensor was $2.625 \mathrm{~nm} / \mathrm{pH}$. 


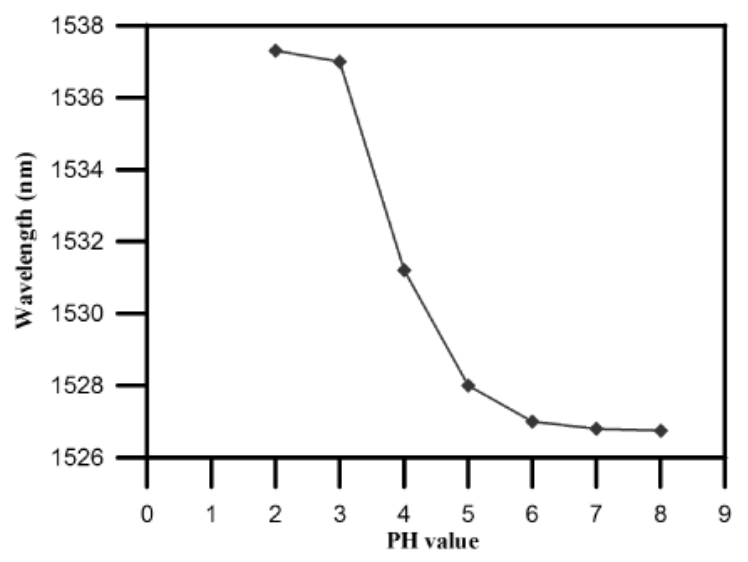

Fig. 7. Wavelength shift with various $\mathrm{pH}$ buffer solutions.

\section{Conclusions}

The optical fiber long-period grating is not only a highly sensitive refractive sensor, it can also be used to determine the $\mathrm{pH}$ of a solution by applying a $\mathrm{pH}$-sensitive hydrogel coating. CLPFG coated with PVA-PAA demonstrated significantly sensitive measurements between $\mathrm{pHs} 2$ and 6 , with a sensitivity of at least $2.625 \mathrm{~nm} / \mathrm{pH}$.

\section{Acknowledgements}

This work was funded by the National Science Council (grant number NSC-1002628-E-151-002-MY3).

\section{References}

1 M. Vaziri and C. L. Chen: J. Lightwave Technol. 10 (1992) 836.

2 S. K. Abi Kaed Bey, T. Sun and K. T. V. Grattan: Sens. Actuators, A 141 (2008) 314.

3 C. Kin Seng, L. Yunqi, N. Mei Nar and D. Xiaoyi: Electron. Lett. 36 (2000) 966.

4 M. Jiang, A. P. Zhang, Y. C. Wang, H. Y. Tam and S. He: Opt. Express 17 (2009) 17976.

5 K. Wang, D. Klimov and Z. Kolber: Opt. Eng. 48 (2009) 034401.

6 Y. H. Jeon, J. J. Kwon and B. H. Lee: J. Opt. Soc. Korea. 10 (2006) 28.

7 C. C. Chiang, H. J. Chang and J. S. Kuo: J. Micro/Nanolith. MEMS MOEMS 9 (2010) 033007. 